\title{
Prótesis de recubrimiento en húmero proximal: ¿Cuál es la indicación hoy en día?
}

\author{
Sánchez-Hernández J, * Jiménez I, ${ }^{*}$ Kiimetoglou D, * Muratore G,* Medina J, * García AM* \\ Hospital Universitario Insular de Gran Canaria
}

RESUMEN. Introducción: Los resultados funcionales de las prótesis de superficie en el húmero proximal son aceptables, pero con grandes diferencias en la tasa y causa de revisión según las diferentes series. El objetivo de nuestro trabajo fue analizar los resultados clínicos obtenidos en nuestro centro y tratar de definir al paciente ideal para este implante. Material y métodos: Estudio retrospectivo de 19 casos. Seguimiento de 31 (12-61) meses. La cirugía se indicó en casos de artrosis primaria o secundaria. Se analizaron datos demográficos, escala de Constant normalizada, cuestionario DASH, complicaciones y satisfacción. Hubo tres pérdidas durante el seguimiento por fallecimiento sin relación con la cirugía. Resultados: Edad media de 56 (25-80) años. La puntuación en la escala de Constant normalizada fue 73 (23-104) puntos y en el cuestionario DASH 31 (7-84) puntos. Noventa y cuatro por ciento de los pacientes retomaron sus actividades de ocio. En siete casos se objetivó radiológicamente colocación en varo del implante generando un conflicto inferior en la glena. Se produjo una capsulitis adhesiva resuelta de forma conservadora, tres reintervenciones por usura glenoidea sintomática y una por dolor incontrolable. Los pacientes sin lesión del manguito rotador presentaron mejor puntuación en los cuestionarios funcionales. Todos los pacientes refirieron estar satisfechos con la mejoría sintomática respecto a la situación previa. Conclusiones: Los resultados fun-
ABSTRACT. Introduction: Data published in the literature about humeral resurfacing prostheses are not conclusive with good functional results but with large differences in the revision rate. The aim of our study was to evaluate the clinical and functional outcomes in patients operated at our center. Material and methods: Retrospective study of 19 cases in 18 patients. Follow-up of 31 (12-61) months. Surgery was indicated in cases of primary or secondary osteoarthritis. Demographic data, Constant scale, DASH questionnaire, complications and satisfaction were analyzed. Three patients were lost to follow-up due to death unrelated to surgery. Results: Mean age of 56 (2580) years. Constant normalized of 73 (23-104) points. DASH questionnaire of 31 (7-84) points. Ninety four percent of the patients resumed their recreational activities and $81 \%$ sports activities. In seven cases, inferior conflict in the glenoid with varus implant was observed radiologically. There were five complications; a conservatively resolved capsulitis, three reinterventions due to symptomatic inferior glenoid usury and another to uncontrollable pain in a conservative way. Patients without rotator cuff lesion presented better scores on the functional questionnaires. All patients were satisfied with the symptomatic improvement over the previous situation and would be operated again if necessary. Conclusions: The functional outcomes reported are similar to those previously published

Nivel de evidencia: IV

\footnotetext{
* Departamento de Cirugía Ortopédica y Traumatología. Hospital Universitario Insular de Gran Canaria. Las Palmas de Gran Canaria, España.

Dirección para correspondencia:

Juan Sánchez-Hernández

Departamento de Cirugía Ortopédica y Traumatología.

Hospital Universitario Insular de Gran Canaria.

Avenida Marítima del Sur s/n, Las Palmas, CP 35016, Las Palmas de Gran Canaria, España.

E-mail: juansanchezhdez@gmail.com
}

Este artículo puede ser consultado en versión completa en http://www.medigraphic.com/actaortopedica 
cionales obtenidos son similares a los publicados previamente. Creemos que la prótesis de superficie debe ser considerada una opción en los casos de artrosis primaria o secundaria sin lesión del manguito rotador, independientemente de la edad.

Palabras clave: Artroplastía, hombro, húmero, prótesis, recubrimiento.

\section{Introducción}

La artroplastía de hombro comenzó a desarrollarse como una opción de tratamiento para pacientes con artrosis glenohumeral a principios de la década de 1950 con la publicación de los primeros diseños anatómicos. ${ }^{1}$ Tras varias décadas de trabajo, investigación y diseño de nuevos implantes, la artroplastía de hombro se ha convertido en el tratamiento quirúrgico estándar de la artrosis consiguiendo importantes mejoras en la función y calidad de vida de los pacientes con una adecuada supervivencia del implante. . $, 3,4,5^{-}$

En los años 70 se realizó el primer procedimiento de superficie en el hombro utilizando un implante de cadera ${ }^{3} \mathrm{y}$ desde entonces se han desarrollado implantes específicos para el húmero proximal. ${ }^{6}$

Entre las ventajas que ofrecen estos implantes sin vástago es importante destacar la conservación de stock óseo, la baja prevalencia de fracturas periprotésicas, menor tiempo quirúrgico y una cirugía de revisión más sencilla cuando sea necesario ${ }^{6,7}$ y además, permite restaurar las relaciones anatómicas nativas del húmero proximal. ${ }^{7,8}$ Por el contrario, el principal inconveniente parece ser la alta tasa de revisiones que se ha publicado en algunas series. ${ }^{9}$

Los resultados funcionales son equiparables a las prótesis parciales con vástago,${ }^{10}$ pero parecen ser inferiores a la artroplastía total anatómica. ${ }^{9}$

Pese a las ventajas teóricas existentes, en la práctica clínica actual la prótesis de superficie parece haber quedado relegada a un segundo plano en pro de la prótesis anatómica con componente glenoideo y de la artroplastía invertida.

El objetivo principal de nuestro trabajo fue analizar los resultados clínicos obtenidos en los pacientes en los que se había implantado una prótesis de hombro sin vástago tipo resurfacing y con base en nuestros resultados, tratar de definir al paciente ideal para este tipo de implante.

\section{Material y métodos}

Tras obtener la aprobación de la Unidad de Formación Continuada y Docencia de nuestro centro, se realizó un estudio de cohorte retrospectiva de 19 artroplastías de hombro de superficie en 18 pacientes intervenidos quirúrgicamente en nuestro centro durante el período 2011-2015. Se and, based on them, we believe that the resurfacing prosthesis is an option to be considered in cases of primary or secondary osteoarthritis when there is no rotator cuff injury, regardless of age.

Key words: Arthroplasty, shoulder, humerus, prosthesis, resurfacing.

revisaron las historias clínicas y se evaluó nuevamente a los pacientes.

La técnica quirúrgica se indicó en aquellos pacientes a los que se les diagnosticó de omartrosis primaria, artrosis secundaria a necrosis avascular, artropatía por lesión del manguito rotador o artrosis postraumática una vez agotado el tratamiento conservador incluyendo tratamiento farmacológico, tratamiento físico rehabilitador y viscosuplementación.

El diagnóstico fue clínico y radiológico definiendo la artrosis glenohumeral como aquella enfermedad degenerativa de la articulación entre la cabeza humeral y la cavidad glenoidea de la escápula (glenoides), en la que se produce una pérdida del cartílago que recubre las superficies articulares con desaparición del espacio articular, así como un endurecimiento de las superficies óseas con formación de osteofitos.

Los criterios de inclusión para el trabajo fueron (1) el diagnóstico de artrosis primaria o secundaria de hombro, (2) la no respuesta a tratamiento conservador, (3) tratamiento quirúrgico siendo el implante una prótesis de superficie y (4) con un seguimiento clínico mínimo de un año tras la cirugía.

\section{Técnica quirúrgica}

En todos los casos la anestesia fue general, se colocó al paciente en posición de silla de playa, se realizó un abordaje deltopectoral, abordaje articular disecando transversalmente las fibras del músculo subescapular o desinsertando cuidadosamente el tendón del subescapular, se realizó el fresado del cartílago articular y hueso subcondral y se colocó el implante SMRTM Resurfacing (Lima Corporate, Italia, 2004) eligiendo el tamaño del implante según las recomendaciones de la técnica quirúrgica de la casa comercial. Se reanclaron las inserciones tendinosas utilizando una sutura no reabsorbible trenzada (Ethibond Excel ${ }^{\circledR}$ 2, Ethicon, Johnson \& Johnson, EEUU) y se realizó un cierre por planos.

Se mantuvo al paciente con un cabestrillo en el postoperatorio inmediato y se autorizaron, desde el día siguiente a la intervención, ejercicios de péndulo y movilización pasiva asistida por el otro miembro. Se indicó retirar de manera progresiva el cabestrillo a las dos semanas de la intervención y se remitió al paciente a rehabilitación, con un comienzo de la misma a las seis semanas aproximadamente. 
Todos los pacientes fueron intervenidos por un facultativo integrante de la Unidad de Cirugía de la Mano y Miembro Superior de nuestro centro.

Además de los datos demográficos y comorbilidades, se registró el diagnóstico, tiempo de seguimiento, escala funcional normalizada de Constant y Murley, ${ }^{11,12}$ discapacidad autopercibida mediante la versión española del cuestionario Disabilities of the Arm, Shoulder and Hand (DASH) ${ }^{13}$ una encuesta cerrada de satisfacción al final del seguimiento, complicaciones radiológicas y complicaciones clínicas. Además, se evaluaron los resultados teniendo en cuenta el diagnóstico preoperatorio.

Hubo tres pérdidas durante el seguimiento por fallecimiento del paciente sin relación directa con la cirugía en ningún caso.

Este trabajo de cohorte retrospectiva no precisó de autorización por parte del Comité de Ensayos Clínicos de nuestro centro. Todos los autores participaron en el diseño, evaluación de los pacientes, recolección de datos y redacción del trabajo.

\section{Resultados}

Se evaluó un total de 16 casos. La edad media de nuestros pacientes en el momento de la cirugía fue de 56 años (rango, 25-80 años) y el seguimiento clínico postoperatorio fue de 31 meses por término medio (rango: 12-61 meses).

La mitad de los pacientes fueron mujeres. Cincuenta por ciento de nuestros pacientes presentaba hipertensión arterial, cuatro de los 16 casos eran dislipémicos, dos eran diabéticos, dos estaban diagnosticados de depresión, uno de espondilitis anquilosante, uno de enfermedad de Paget, uno de lupus eritematoso sistémico, uno de hipotiroidismo y uno de epilepsia.

El lado intervenido fue el derecho en $69 \%$ de los casos. La actividad laboral preoperatoria fue considerada como ligera (administrativo, educación) en tres casos, moderada (hostelería, ama de casa, mantenimiento) en 11 casos y pesada (construcción, agricultura) en dos casos.
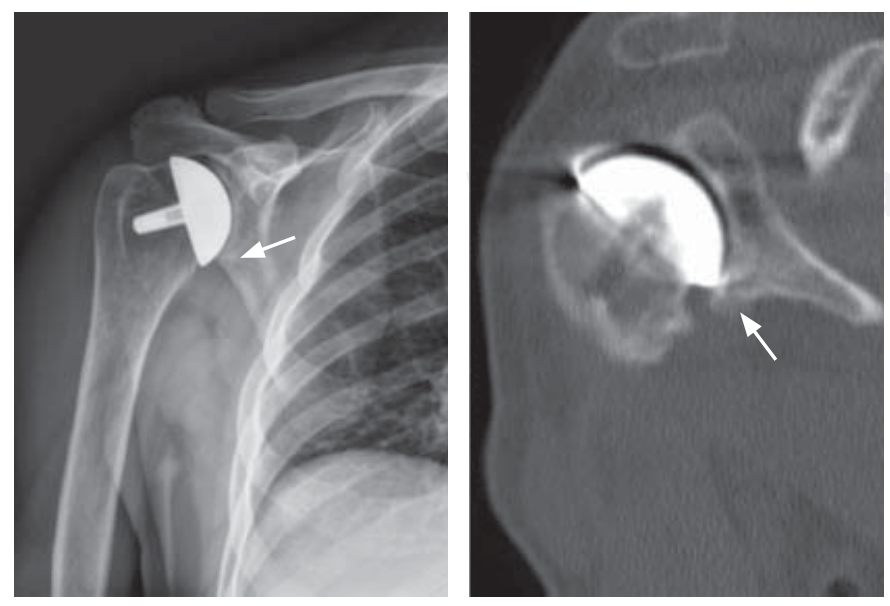

El diagnóstico fue omartrosis primaria en 10 casos $(62.5 \%)$, artrosis secundaria a rotura del manguito rotador en tres casos, artrosis secundaria a necrosis avascular en dos casos y artrosis secundaria a fractura del húmero proximal en un caso.

La puntuación media al final del seguimiento en la escala de Constant normalizada fue de 73 puntos (desviación estándar 22.7; rango 21-104). Según esta escala, el resultado fue excelente en seis casos, bueno en cuatro, regular en tres y malo en tres.

La puntuación media en el cuestionario DASH fue de 31 puntos (desviación estándar 24.4; rango 7-84).

No mostraba dolor $62.5 \%$ de los pacientes o éste era leve al final del seguimiento, evaluado según el subapartado para el dolor de la escala de Constant o presentaba una puntuación menor de cinco puntos en la escala visual analógica (EVA) para dolor.

Quince de los 16 pacientes retomaron sus actividades de ocio en un tiempo medio de 3.5 semanas y 13 de los 16 pacientes retomaron sus actividades deportivas a las nueve semanas de la intervención por término medio.

En siete de los 16 casos observamos una disposición del implante en varo generando un conflicto inferior en la glena (Figura 1), si bien esta complicación radiológica únicamente tuvo repercusión clínica en tres casos.

Hubo una capsulitis adhesiva postoperatoria resuelta de forma conservadora con un programa de rehabilitación y estiramiento capsular. En tres casos se produjo una usura glenoidea inferior sintomática que requirió reintervención. En un caso intervenido por lesión del manguito rotador hubo que recambiar el implante a una prótesis tipo CTA (Cuff Tear Arthropathy) por dolor incontrolable de forma conservadora.

Al analizar los resultados en función del diagnóstico preoperatorio, observamos que los resultados variaron notablemente en función del estado del manguito rotador.

En los casos intervenidos por una entidad nosológica distinta a lesión del manguito, la puntuación en la escala de Constant normalizada fue de 79 puntos por término medio, mientras que la puntación en los casos de lesión del manguito rotador fue de 55 puntos $(\mathrm{p}=0.07)$. La pun-

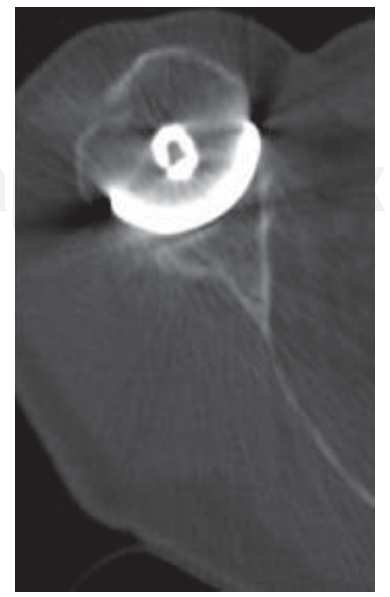

Figura 1:

Implante posicionado en varo (anormalmente dirigido hacia la línea media del cuerpo). Flecha: conflicto inferior en la glena. 
tuación en el cuestionario DASH fue de 27 puntos en los casos de manguito rotador íntegro por 49 en los casos que presentaban una lesión del manguito $(\mathrm{p}=0.09)$.

$\mathrm{Al}$ analizar los resultados en función de la edad, observamos que los pacientes mayores de 60 años obtuvieron una puntuación promedio en la escala de Constant de 79.7 puntos, en tanto que los menores de 60 años obtuvieron una puntuación de 68.3. En el cuestionario DASH, de forma similar, los mayores de 60 años obtuvieron una puntuación de 19.8 puntos por 40.2 en el caso de los menores de 60 años. Estos resultados no fueron estadísticamente significativos (escala de Constant $\mathrm{p}=0.9$; cuestionario DASH $\mathrm{p}=0.47$.

No hubo diferencias en los resultados en función del sexo.

Todos los pacientes refirieron estar satisfechos con la mejoría clínica respecto a la situación previa y se operarían de nuevo si fuera necesario.

\section{Discusión}

La prótesis de superficie ofrece múltiples ventajas para el cirujano y para el paciente. La resección ósea es menor manteniendo la cabeza humeral, evitando crear un nuevo ángulo cabeza-diáfisis y consiguiendo reproducir la geometría del húmero proximal de forma más precisa. ${ }^{7,8,14,15} \mathrm{Se}$ evita la penetración del canal medular, el riesgo de fractura periprotésica es bajo y los resultados funcionales son al menos comparables con los obtenidos por las prótesis con vástago intramedular. ${ }^{10,16,17}$ Para el paciente la rehabilitación postoperatoria es más sencilla tras la prótesis de superficie, ${ }^{18}$ teóricamente se mantiene la retroalimentación propioceptiva, lo que puede conducir a una mejor función ${ }^{4,17}$ y la satisfacción postoperatoria es similar a la obtenida con la artroplastía total. . $^{3,5,5,18,19,20}$

No todo es ventajas. Técnicamente la exposición de la cabeza humeral no es fácil pudiendo provocar una excesiva anteversión y/o posicionamiento en varo del implante. ${ }^{14}$ Además, en casos con gran deformidad ósea por los osteofitos, la elección del tamaño del implante requiere gran experiencia; en la serie publicada por Smith y cols. ${ }^{21}$ sólo 28 de las 50 prótesis evaluadas tenían un tamaño apropiado y este sobredimensionamiento puede condicionar un desgaste progresivo de la glena, como se ha publicado en varios trabajos. $^{21,22,23}$

La tasa de aflojamiento parece mayor que con las prótesis con vástago ${ }^{18,24,25} \mathrm{y}$ el riesgo de fractura del cuello o el colapso de la cabeza humeral serían posibles complicaciones añadidas como ocurre en la resuperficialización de la cadera. $^{25,26}$

Utilizamos la escala de Constant normalizada para valorar la función obteniendo un valor promedio de 73 puntos. Al analizar los resultados en función del diagnóstico preoperatorio, observamos que en los casos intervenidos por lesión del manguito rotador la puntuación fue de 55 puntos, mientras que en los casos intervenidos por otra causa, la puntuación alcanzó 79 puntos.
En el cuestionario de discapacidad DASH el resultado promedio fue de 31 puntos. De igual forma, al analizar los resultados en función del diagnóstico preoperatorio observamos que en los casos de lesión del manguito el resultado fue de 49 puntos, en tanto que en los casos intervenidos por otra causa el resultado fue de 27.

Se analizaron los resultados en función de las comorbilidades, sin encontrar diferencias significativas en función de las mismas.

Con base en estos resultados, la causa principal de obtener un mal resultado funcional en nuestra serie fue la indicación del implante en casos con lesión del manguito rotador. Estos resultados no mostraron significancia estadística, aunque sí una clara tendencia a la misma.

Los resultados funcionales obtenidos son similares a otros trabajos publicados previamente con una puntuación al final del seguimiento en la escala de Constant que oscila entre 50 y 80 puntos (Tabla 1). 18,22,27,28,29

La tasa de revisión es alta. En nuestra serie fue de cuatro de 16 pacientes, similar a otros trabajos publicados. En el estudio de Rasmussen y cols. ${ }^{9}$ basado en el registro danés, la tasa de reintervención fue de $9.9 \%$, en el registro neozelandés de $10 \%$ y en el noruego alcanzó $17 \%$. Además, otros autores han publicado cifras de reintervención que ascienden hasta $18.5 \%{ }^{23,30}$

En nuestra serie, la necesidad de revisión la atribuimos a una mala indicación de implante de forma preoperatoria (lesiones del manguito rotador) y errores técnicos relacionados con la colocación de la prótesis. ${ }^{22}$ En siete de los 16 casos observamos un conflicto inferior en la glena por un implante posicionado en varo, si bien este hallazgo no generó un mal resultado funcional per se, tal es así que el paciente con mejor resultado funcional (Constant 90 puntos, DASH 7.7 puntos) presenta la prótesis en varo (Figura 2).

El resultado percibido por el paciente es bueno. En nuestra serie todos los pacientes refirieron encontrarse mejor, hallazgos similares a otros trabajos en los que la satisfacción alcanza $95 \%$ en una serie de 75 hombros, $94 \%$ en una serie de 103 hombros o $90 \%$ en una serie de 79 hombros. ${ }^{17,18,27}$

\begin{tabular}{|c|c|c|c|}
\hline \multicolumn{4}{|c|}{$\begin{array}{l}\text { Tabla 1: Resumen de las principales } \\
\text { series publicadas en la bibliografía. }\end{array}$} \\
\hline & $\begin{array}{l}\text { Tamaño } \\
\text { muestral }\end{array}$ & $\begin{array}{l}\text { Seguimiento } \\
\text { (meses) }\end{array}$ & $\begin{array}{c}\text { Escala de } \\
\text { Constant } \\
\text { (promedio) }\end{array}$ \\
\hline Levy y Copeland ${ }^{27}$ & 30 & 24 & 58.0 \\
\hline Levy et al. ${ }^{30}$ & 42 & 174 & 71.8 \\
\hline Thomas et al. ${ }^{31}$ & 48 & 34 & 54.0 \\
\hline Mullett et al. ${ }^{29}$ & 29 & 54 & 77.0 \\
\hline Alizadehkhaiyat et al. ${ }^{20}$ & 102 & 48 & 52.2 \\
\hline Lebon et al. ${ }^{19}$ & 41 & 40 & 77.0 \\
\hline Geervliet et al. ${ }^{32}$ & 47 & 24 & 79.0 \\
\hline Mansat et al. ${ }^{22}$ & 64 & 36 & 68.0 \\
\hline Merolla et al. ${ }^{33}$ & 60 & 44 & 65.0 \\
\hline Glanzmann et al. ${ }^{34}$ & 31 & 24 & 70.6 \\
\hline Smith et al..$^{21}$ & 50 & 30 & 73.0 \\
\hline
\end{tabular}




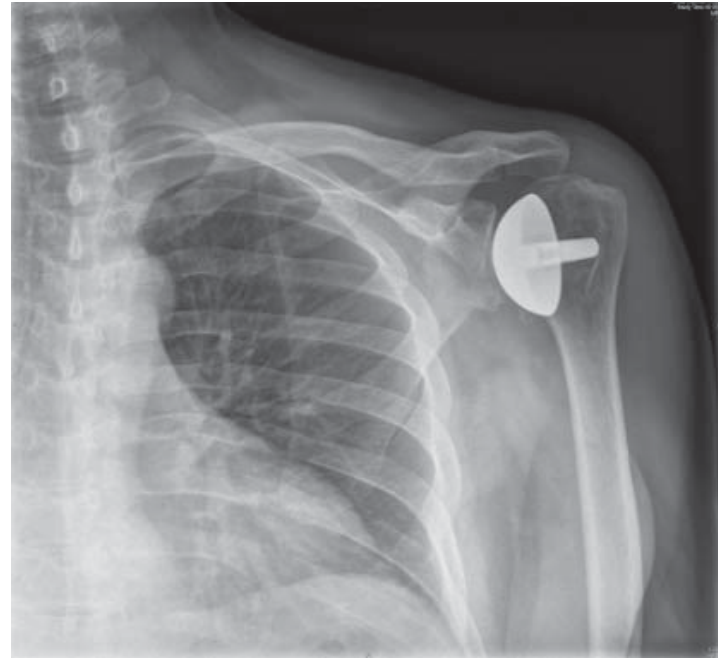

Figura 2: Implante en posición horizontal que no tuvo repercusión funcional.

En nuestra serie obtuvimos mejores resultados en los pacientes mayores de 60 años, aunque estos resultados no fueron estadísticamente significativos. Elegimos los 60 años de forma arbitraria, si bien la definición de paciente joven está cuestionada habiéndose sugerido en varios trabajos los 5060 años de edad. ${ }^{2,20,30}$ Creemos que definir un límite de edad puede ser de gran interés, ya que en pacientes jóvenes, con probable mayor demanda funcional, la prótesis de superficie parece obtener peores resultados.

Por último, de acuerdo con lo publicado previamente, el resultado funcional parece ser mejor en los casos de artrosis primaria frente a secundaria a procesos sépticos o artropatía del manguito. ${ }^{27,29}$ En nuestra serie no disponemos de un tamaño muestral suficiente para poder valorar este aspecto.

Basándonos en nuestros resultados, podemos definir al paciente ideal para someterse a una prótesis de superficie de hombro como un varón o mujer mayor de 60 años que presenta artrosis glenohumeral primaria con integridad del manguito rotador.

Por otro lado, también podríamos definir al paciente en el que no debemos colocar este tipo de implante como un varón o mujer menor de 60 años que presenta omartrosis secundaria a una rotura del manguito rotador.

Nuestro estudio tiene limitaciones. Se trata de una serie de cohorte retrospectiva y carece de grupo control. No disponemos de datos funcionales preoperatorios. El tamaño muestral es pequeño y el seguimiento es corto en algunos casos para poder analizar las posibles complicaciones a largo plazo.

Consideramos que la prótesis de superficie en el tratamiento de la artrosis glenohumeral puede ser una opción de tratamiento adecuada para conseguir un buen resultado funcional en los casos de artrosis primaria o secundaria, siempre que el manguito rotador se encuentre íntegro.

Conflicto de Intereses: Los autores declaran que no existe conflicto de intereses.
Bibliografía

1. Krueger FJ. A vitallium replica arthroplasty on the shoulder; a case report of aseptic necrosis of the proximal end of the humerus. Surgery. 1951; 30(6): 1005-11.

2. Johnson MH, Paxton ES, Green A. Shoulder arthroplasty options in young ( $<50$ years old) patients: review of current concepts. J Shoulder Elbow Surg. 2015; 24(2): 317-25.

3. Edwards TB, Kadakia NR, Boulahia A, Kempf JF, Boileau P, Némoz C, et al. A comparison of hemiarthroplasty and total shoulder arthroplasty in the treatment of primary glenohumeral osteoarthritis: results of a multicenter study. J Shoulder Elbow Surg. 2003; 12(3): 207-13.

4. Gartsman GM, Roddey TS, Hammerman SM. Shoulder arthroplasty with or without resurfacing of the glenoid in patients who have osteoarthritis. J Bone Joint Surg Am. 2000; 82(1): 26-34.

5. Radnay CS, Setter KJ, Chambers L, Levine WN, Bigliani LU, Ahmad CS. Total shoulder replacement compared with humeral head replacement for the treatment of primary glenohumeral osteoarthritis: a systematic review. J Shoulder Elbow Surg. 2007; 16(4): 396-402.

6. Rai P, Davies O, Wand J, Bigsby E. Long-term follow-up of the Copeland mark III shoulder resurfacing hemi-arthroplasty. J Orthop. 2015; 13(1): 52-6.

7. Burgess DL, McGrath MS, Bonutti PM, Marker DR, Delanois RE, Mont MA. Shoulder resurfacing. J Bone Joint Surg Am. 2009; 91(5): 1228-38.

8. Hammond G, Tibone JE, McGarry MH, Jun BJ, Lee TQ Biomechanical comparison of anatomic humeral head resurfacing and hemiarthroplasty in functional glenohumeral positions. $J$ Bone Joint Surg Am. 2012; 94(1): 68-76.

9. Rasmussen JV, Polk A, Sorensen AK, Olsen BS, Brorson S. Outcome, revision rate and indication for revision following resurfacing hemiarthroplasty for osteoarthritis of the shoulder: 837 operations reported to the Danish Shoulder Arthroplasty Registry. Bone Joint J. 2014; 96-B(4): 519-25.

10. Levy O. Shoulder resurfacing: is it really as good as total shoulder replacement? Curr Orthop Pract. 2012; 23: 2-9.

11. Constant CR, Murley AH. A clinical method of functional assessment of the shoulder. Clin Orthop Relat Res. 1987; (214): 160-4.

12. Katolik LI, Romeo AA, Cole BJ, Verma NN, Hayden JK, Bach BR. Normalization of the Constant score. J Shoulder Elbow Surg. 2005; 14(3): 279-85.

13. Rosales RS, Delgado EB, Díez de la Lastra-Bosch I. Evaluation of the Spanish version of the DASH and carpal tunnel syndrome healthrelated quality-of-life instruments: cross-cultural adaptation process and reliability. J Hand Surg Am. 2002; 27(2): 334-43.

14. Deladerrière JY, Szymanski C, Vervoort T, Budzik JF, Maynou C. Geometrical analysis results of 42 resurfacing shoulder prostheses: A CT scan study. Orthop Traumatol Surg Res. 2012; 98(5): 520-7.

15. Haines JF, Trail IA, Nuttall D, Birch A, Barrow A. The results of arthroplasty in osteoarthritis of the shoulder. J Bone Joint Surg Br. 2006; 88(4): 496-501.

16. Jensen KL. Humeral resurfacing arthroplasty: rationale, indications, technique, and results. Am J Orthop (Belle Mead NJ). 2007; 36(12 Suppl 1): 4-8.

17. Levy O, Copeland SA. Cementless surface replacement arthroplasty of the shoulder. 5- to 10-year results with the Copeland mark-2 prosthesis. J Bone Joint Surg Br. 2001; 83(2): 213-21.

18. Pritchett JW. Long-term results and patient satisfaction after shoulder resurfacing. J Shoulder Elbow Surg. 2011; 20(5): 771-7.

19. Lebon J, Delclaux S, Bonnevialle N, Rongières $\mathrm{M}$, Bonnevialle $\mathrm{P}$, Mansat P; French Society for Shoulder, Elbow (SOFEC). Stemmed hemiarthroplasty versus resurfacing in primary shoulder osteoarthritis: a single-center retrospective series of 78 patients. Orthop Traumatol Surg Res. 2014; 100(6 Suppl): S327-32.

20. Alizadehkhaiyat O, Kyriakos A, Singer MS, Frostick SP. Outcome of Copeland shoulder resurfacing arthroplasty with a 4-year mean follow-up. J Shoulder Elbow Surg. 2013; 22(10): 1352-8.

21. Smith T, Gettmann A, Wellmann M, Pastor F, Struck M. Humeral surface replacement for osteoarthritis. Acta Orthop. 2013; 84(5): 468-72. 
22. Mansat $\mathrm{P}$, Coutié AS, Bonnevialle N, Rongières M, Mansat M, Bonnevialle P. Resurfacing humeral prosthesis: do we really reconstruct the anatomy? J Shoulder Elbow Surg. 2013; 22(5): 612-9.

23. Soudy K, Szymanski C, Lalanne C, Bourgault C, Thiounn A, Cotten A, et al. Results and limitations of humeral head resurfacing: 105 cases at a mean follow-up of 5 years. Orthop Traumatol Surg Res. 2017; 103(3): 415-20.

24. Rydholm U, Sjögren J. Surface replacement of the humeral head in the rheumatoid shoulder. J Shoulder Elbow Surg. 1993; 2(6): 286-95.

25. Jónsson E, Lidgren L, Mjöberg B, Rydholm U, Selvik G. Humeral cup fixation in rheumatoid shoulders. Roentgen stereophotogrammetry of 12 cases. Acta Orthop Scand. 1990; 61(2): 116-7.

26. Pritchett JW. Curved-stem hip resurfacing: minimum 20-year followup. Clin Orthop Relat Res. 2008; 466(5): 1177-85.

27. Levy O, Copeland SA. Cementless surface replacement arthroplasty (Copeland CSRA) for osteoarthritis of the shoulder. J Shoulder Elbow Surg. 2004; 13(3): 266-71.

28. Al-Hadithy N, Domos P, Sewell MD, Naleem A, Papanna MC, Pandit R. Cementless surface replacement arthroplasty of the shoulder for osteoarthritis: results of fifty Mark III Copeland prosthesis from an independent center with four-year mean follow-up. J Shoulder Elbow Surg. 2012; 21(12): 1776-81.
29. Mullett H, Levy O, Raj D, Even T, Abraham R, Copeland SA Copeland surface replacement of the shoulder. Results of an hydroxyapatite-coated cementless implant in patients over 80 years of age. J Bone Joint Surg Br. 2007; 89(11): 1466-9.

30. Levy O, Tsvieli O, Merchant J, Young L, Trimarchi A, Dattani R, et al. Surface replacement arthroplasty for glenohumeral arthropathy in patients aged younger than fifty years: results after a minimum tenyear follow-up. J Shoulder Elbow Surg. 2015; 24(7): 1049-60.

31. Thomas SR, Wilson AJ, Chambler A, Harding I, Thomas M. Outcome of Copeland surface replacement shoulder arthroplasty. $J$ Shoulder Elbow Surg. 2005; 14(5): 485-91.

32. Geervliet $P$, van den Bekerom M, Spruyt P, Curvers M, Visser C, van Noort A. Short-term results of the global C.A.P. uncemented resurfacing shoulder prosthesis. Orthopedics. 2014; 37(1): 42-7.

33. Merolla G, Bianchi P, Lollino N, Rossi R, Paladini P, Porcellini G. Clinical and radiographic mid-term outcomes after shoulder resurfacing in patients aged 50 years old or younger. Musculoskelet Surg. 2013; 97 Suppl 1: 23-9.

34. Glanzmann M, Kolling C, Schwyzer HK, Flury M, Audigé L. Radiological and functional 24-month outcomes of resurfacing versus stemmed anatomic total shoulder arthroplasty. Int Orthop. 2017; 41(2): $375-84$. 